\title{
Number 1
}

\section{Asthma}

\author{
Richard Leigh MBChB FCPSA and Staff \\ Firestone Regional Chest \& Allergy Unit, St Joseph's Hospital, Hamilton, Ontario
}

\begin{abstract}
What is asthma?
Asthma is a common condition affecting the lungs that causes a variety of variable symptoms including shortness of breath, chest tightness, wheezy breathing, cough, and production of sputum or phlegm. The big difference between asthma and other obstructive lung conditions (chronic bronchitis, emphysema) is the great variability in symptoms from day to day, week to week and season to season; symptoms may not be present for long periods and can respond, often dramatically, to treatment. Also, asthma does not lead to emphysema or heart disease. Thus, although asthma needs to be taken seriously, it should not cause alarm because the aim of treatment is to render the patient completely free of symptoms.
\end{abstract}

\section{What causes asthma?}

The symptoms of asthma are caused by a special type of inflammation in the walls of the airways (bronchial tubes), causing swelling, mucus production and tightening of the small muscles in the walls; these processes lead to narrowing of the airways. Basically, the inflammation has two main causes - allergic reactions to inhaled particles in the air and what is termed 'airway reactivity'. Commonly, both factors are present but to a variable extent in different subjects. In some patients, allergy may be the more important cause, while in other patients, it may be the airway reactivity. The extremes of this 'spectrum' are represented by seasonal asthma due to ragweed allergy and asthma occurring on exercise or in very cold air.

\section{What allergies cause asthma?}

There are literally hundreds of particles to which allergic inflammation may develop. Some common allergens include plant pollens (trees, grasses, ragweed, etc), animal dander (cats and dogs), insects (house dust mites) and molds. Some people develop asthma as part of a general allergic reaction to things they have eaten such as shellfish, peanuts, chocolate and drugs (such as acetylsalicylic acid and other arthritis medications, and beta-blockers, which are often used in patients with heart disease and also to treat glaucoma). Chemicals encountered at work may also cause asthma ('occupational asthma'). Allergic factors may be identified by the occurrence of symptoms in relation to exposure, by skin testing and, in some special situations, by specific inhalation studies.

\section{What is 'airway reactivity'?}

In most patients with asthma, the airway muscle and tissue react to stimuli that do not trouble nonasthmatics, including cold air, cigarette smoke, paints and varnish, perfumes, hair sprays and many other strong smelling chemicals. Some patients with reflux of acidic stomach juices into the airways (gastroesophageal reflux) may also develop asthma. Asthma attacks may also be brought on by emotional changes, such as laughing, or be associated with panic attacks. This reactivity, or 'twitchy airways', is identified and measured by a simple inhalation test (methacholine or histamine reactivity), which shows how big a factor this reactivity is in an individual with asthma. 


\section{How is asthma controlled?}

A large amount of research during the past 20 years has amply shown that optimal management of asthma involves a holistic approach that applies a number of measures that are tailored to each individual patient, often termed an 'action plan'. Such measures include the following:

- Patient education.

- Comprehensive assessment of allergies and airway reactivity, with regular reassessment of functional status.

- Avoidance of asthma 'triggers', whether allergens, occupational exposure or other important personal factors.

- Attention to diet, exercise and smoking cessation.

- Corticosteroid inhalers (puffers), used appropriately in an adequate dosage, to reduce airway inflammation.

- Proper use of inhalers that control airway narrowing acutely (beta-agonists).

- Other medications that may help decrease airway inflammation (cromoglycate, leukotriene antagonists).

The aim of treatment is a normal, active lifestyle with complete control of symptoms. Future "Information for Patients" pages will deal with all of these aspects in more detail.

\section{Sources of information on asthma}

Your local lung association (Christmas Seals) has educational material specifically targeted to patients. If you have access to the Internet, there are several Web sites that provide information on asthma, including the following:

- Canadian Lung Association - www.lung.ca; this is an excellent site that contains an A to Z of pulmonary disorders

- Respiratory Therapy Society of Ontario - www.rtso.org

- British Lung Foundation - www.lunguk.org

- American Lung Association - www.lungusa.org

This information should not be used as a substitute for the medical care and advice of your physician. There may be variations in treatment that your physician may recommend based on individual facts and circumstances. This information may be reproduced without permission and shared with patients and their families. 


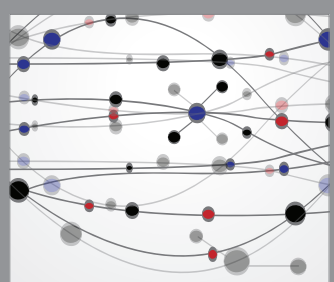

The Scientific World Journal
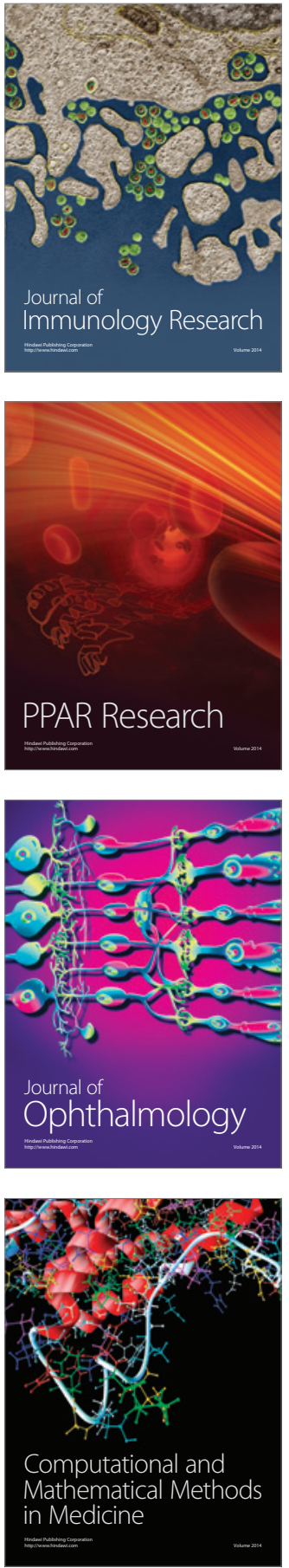

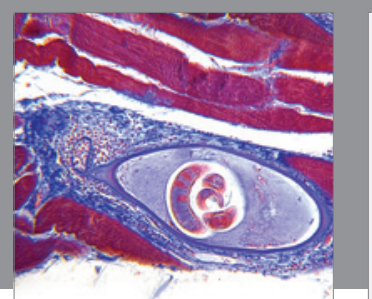

Gastroenterology Research and Practice

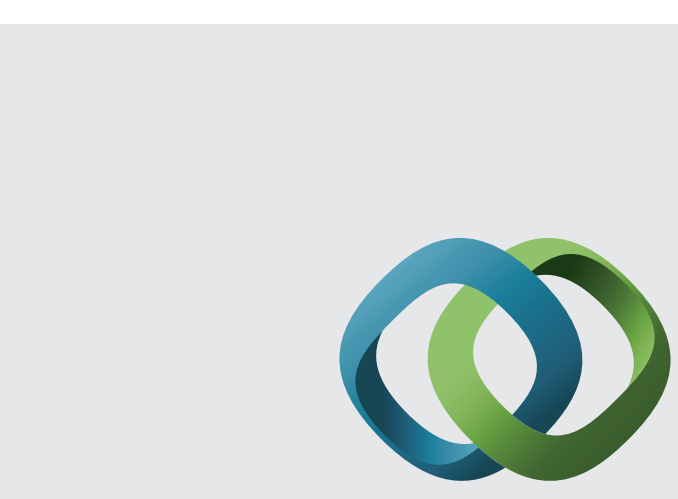

\section{Hindawi}

Submit your manuscripts at

http://www.hindawi.com
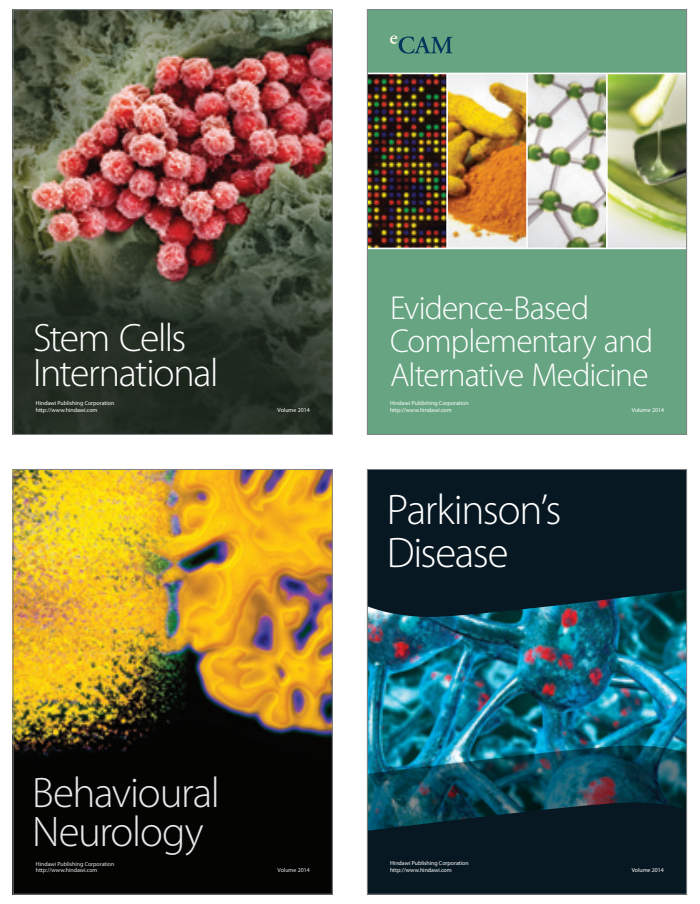
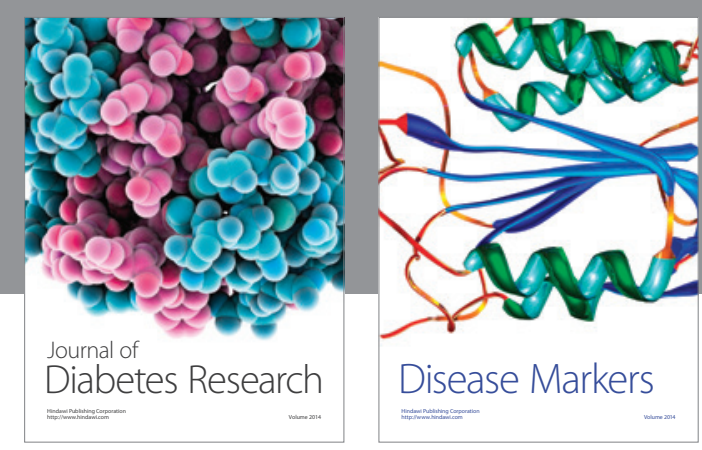

Disease Markers
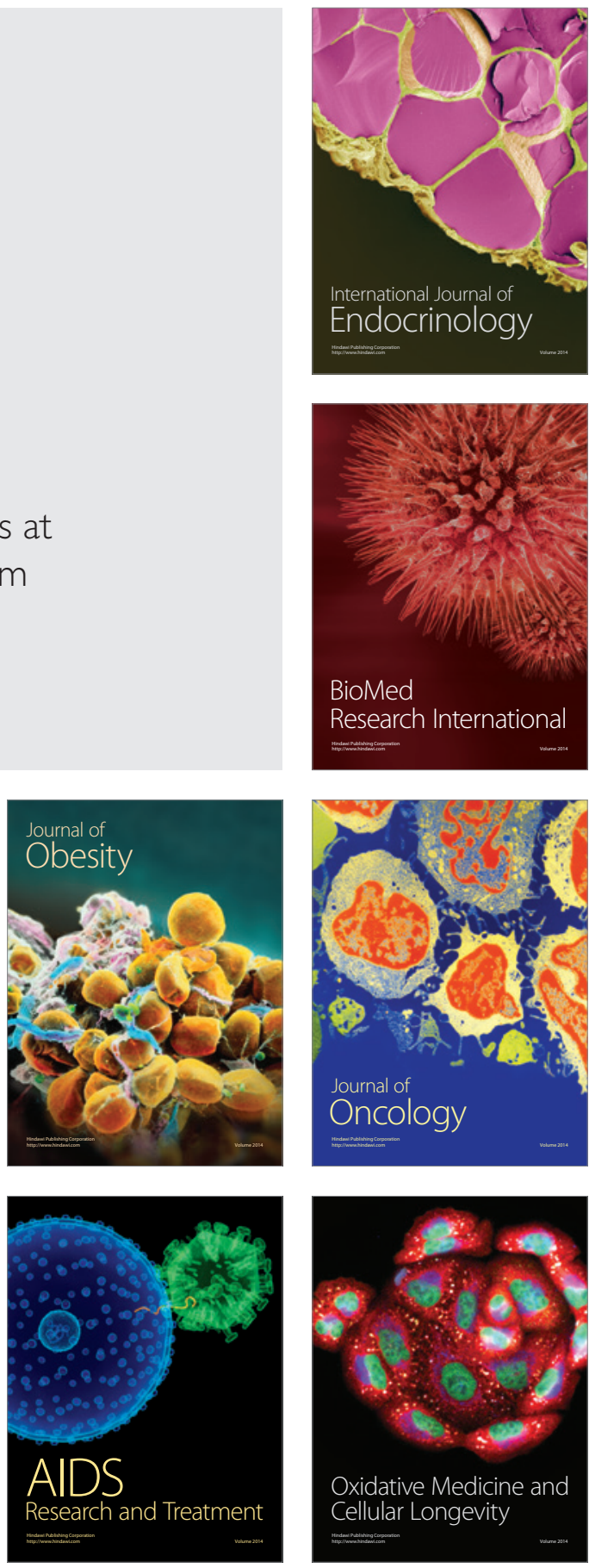